\title{
De candidato a pós-graduando em Design no Brasil: mapeamento da jornada do usuário
}

\author{
From applicant to graduate student in Design in Brazil: mapping the user journey
}

Jéssica Rodrigues Esteves, Larissa Buenaño Ribeiro, Francisco Fialho, Maria Collier de

Mendonça, Richard Perassi, Berenice Santos Gonçalves, Ricardo Triska

pós-graduação, design thinking, jornada do usuário

Ao participarem de um processo seletivo para um Programa de Pós-graduação (PPG), os candidatos realizam uma série de etapas classificatórias que exigem a capacidade de lidar com uma grande carga informacional quanto aos prazos e requisitos. Nesse contexto, a presente pesquisa tem como objetivo identificar como os candidatos buscam informações ao aplicarem para um PPG Stricto Sensu em Design no Brasil. A metodologia de pesquisa combinou três etapas: (I) revisão narrativa, (II) entrevistas qualitativas com coordenadores e candidatos aos PPGs e (III) desenvolvimento dos mapas de empatia e de jornada do usuário. A revisão narrativa incluiu uma breve contextualização sobre o histórico dos PPGs em Design no Brasil, seguida pela apresentação dos referenciais do Design Thinking que guiaram os procedimentos de campo. As entrevistas qualitativas enfocaram a identificação dos critérios que influenciam as decisões dos candidatos. Em seguida, foram criados mapas de empatia e mapas de jornada do usuário para cada um dos candidatos entrevistados. Por fim, foi criado um mapa da jornada do usuário, por meio da identificação de um cenário e das expectativas gerais dos candidatos. Os resultados do estudo apontam orientações para a criação de um serviço digital destinado a informar e auxiliar candidatos e pesquisadores em Design.

graduate studies, design thinking, user journey

When participating in a selection process for a Postgraduate Program (PPG), candidates perform a series of qualifying steps that require the ability to deal with an extensive information load regarding deadlines and requirements. In this context, this research aims to identify how candidates seek information when applying for a PPG Stricto Sensu in Design in Brazil. The research methodology combined three stages: (I) narrative review, (II) qualitative interviews with coordinators and applicants for PPGs, and (III) development of the empathy and user journey maps. The narrative review included a brief contextualization about the history of PPGs in Design in Brazil, followed by the Design Thinking references that guided the field procedures. Qualitative interviews focused on identifying the criteria that influence the decision of applicants. Then, we created an empathy map and user journey map for each of the interviewed applicants. Finally, we created a user journey map of the applicants by identifying a scenario and their expectations. The study results point to guidelines for creating a digital service designed to inform and assist candidates and researchers in Design.

Anais do $10^{\circ} \mathrm{CIDI}$ e $10^{\circ} \mathrm{CONGIC}$

Kelli C.A.S. Smythe, Rafael de Castro Andrade (orgs.)

Sociedade Brasileira de Design da Informação - SBDI

Curitiba | Brasil | 2021
Proceedings of the $10^{\text {th }} \mathrm{CIDI}$ and $10^{\text {th }}$ CONGIC

Kelli C.A.S. Smythe, Rafael de Castro Andrade (orgs.)

Sociedade Brasileira de Design da Informação - SBDI Curitiba | Brazil | 2021 


\section{Introdução}

As pós-graduações stricto sensu compreendem programas de mestrado e doutorado dos quais alunos diplomados em cursos superiores de graduação podem participar dos processos seletivos. Esses processos, por sua vez, são abertos conforme os cronogramas das universidades, obedecendo aos prazos legais publicados em editais anuais ou semestrais, em conformidade com as diretrizes da Coordenação de Aperfeiçoamento de Pessoal de Nível Superior (CAPES).

De acordo com a CAPES, em 1996, existiam 67.820 alunos da pós-graduação no país, sendo 45.622 de mestrado e 22.198 de doutorado. Já em 2003 os números cresceram para 112.237 estudantes de pós-graduação, sendo 66.959 de mestrado acadêmico, 5.065 de mestrado profissional e 40.213 de doutorado. Nos últimos oito anos, o número de cursos de pós-graduação aprovados pela CAPES tem crescido em média $9 \%$ ao ano e o maior número de alunos encontra-se nas áreas de Ciências Humanas e Engenharias, Ciências da Computação e Ciências da Saúde (CAPES, 2021).

Já a pós-graduação em Design, enfoque desta investigação, compõe a área de Arquitetura e Urbanismo e Design ao integrar o sistema CAPES e a grande área de Ciências Sociais Aplicadas (CAPES, 2021). A área das Ciências Sociais Aplicadas pauta-se em princípios de ensino e pesquisa interdisciplinares, portanto, busca ultrapassar a visão disciplinar e setorial de projetos, integrando várias áreas para a compreensão das necessidades da sociedade em sua complexidade, ao possibilitar relações de convergência e de complementaridade de saberes na pesquisa.

O primeiro programa de pós-graduação em Design foi implementado em 1994 na PUC-Rio, a qual também foi a primeira instituição a oferecer o curso de Doutorado em Design, inspirando a organização de outras propostas nas instituições de ensino superior nesta mesma modalidade de formação (Triska, Vela \& Dolzan, 2014).

Atualmente, a subárea de Design soma 24 programas, sendo 4 com mestrado acadêmico (exclusivamente), 12 com mestrado acadêmico e doutorado e 8 com mestrado profissional, um número significativamente baixo, quando comparado às outras áreas de pesquisa tais como Ciência da Computação que possui 88 programas; Engenharias com 129 programas; e Comunicação e Informação possuindo 90 programas (CAPES, 2021).

De acordo com Diniz (2014), o panorama geral de PPGs em Design indica que nem todas as regiões do país oferecem oportunidades para qualificação, porque os programas de pósgraduação ainda concentram-se no eixo Sul e Sudeste. Segundo Diniz (2014) há somente um programa no Norte, um no Centro-Oeste, cinco no Nordeste, oito no Sudeste e oito no Sul; sendo, portanto, necessário integrar os profissionais de pesquisa em Design por meio de esforços coletivos para a expansão desta área.

As demandas regionais, ainda muito setorizadas, têm sido um dos principais desafios para a área, na busca de soluções que atendam uma intensa interlocução acadêmica em nível interregional para a maior popularização da ciência. Neste sentido, a área necessita de iniciativas informacionais para dialogar e estimular projetos que visem equiparar o horizonte de 
oportunidades a todos os candidatos, de maneira ética e isonômica, resgatando uma das facetas que caracterizam o papel social da pós-graduação, a inclusão.

Ao candidatar-se para um processo seletivo em um Programa de Pós-graduação no Brasil, o estudante realiza uma série de etapas, tais como: (I) prova de conhecimentos específicos, (II) prova de proficiência, (III) análise curricular, (III) análise de projeto de pesquisa, (IV) entrevistas.

Dada a complexidade das etapas, os candidatos lidam com uma grande carga informacional quanto aos prazos e requisitos do processo de submissão. Portanto, é essencial que as informações sobre os processos seletivos sejam cuidadosamente organizadas e divulgadas para, mais tarde, serem corretamente interpretadas e compreendidas pela maioria dos indivíduos dentro da audiência pretendida. (Dick, Gonçalves \& Vitorino, 2017, p.3).

Neste contexto, a maneira como os dados e as informações são apresentados possuem um papel importante, pois relacionam-se com o design da informação. Esse, por sua vez, é um domínio em que os conteúdos são visualizados por meio da seleção, do ordenamento, da hierarquização, das conexões e distinções visuais que permitem uma ação eficaz, independente do meio em que tais conteúdos estão disponibilizados (Bonsiepe, 2020). Logo, o design da informação é a informação organizada de forma intencional com o objetivo de criar sentido, proporcionando assim a clareza da informação (Lipton, 2011; Baer \& Vacarra, 2008; Pettersson, 2010)

Para tanto, esta pesquisa de natureza exploratória e caráter qualitativo, tem como objetivo central responder a seguinte questão: como facilitar o acesso à informação sobre os processos seletivos dos programas de pós-graduação em Design em nível inter-regional? Dessa forma, propõem-se identificar como os candidatos buscam informações ao aplicarem para um programa de Pós-graduação em Design no Brasil. Para tal, foram realizadas entrevistas com coordenadores e candidatos junto às duas técnicas de Design Thinking utilizadas durante a fase de imersão (Brown \& Katz, 2011), como o mapa de empatia (Gibbons, 2018a) e o mapa de jornada do usuário (Hanington \& Martin, 2019; Gibbons, 2018b).

\section{Design Thinking}

O Design Thinking é uma abordagem que trata do uso do pensamento do design para a concepção de produtos e soluções para as áreas de marketing, negócios e finanças. Para Lupton (2011) o conceito de Design Thinking refere-se ao processo de ideação, pesquisa, prototipação e interação do usuário. Autores como Osborn (1953) e De Bono (1969) auxiliaram a explicar e popularizar métodos criativos de solução de problemas. Koberg e Bagnall (1972) também contribuíram para a popularização de métodos, apresentando inúmeras formas de utilizar o caminho não linear para a solução de problemas. Recentemente a abordagem tem sido popularizada por Brown e Wyatt (2010) e Kelley (2001) e demais integrantes da IDEO, enfatizando o uso de técnicas abrangentes para enquadrar problemas e gerar soluções com o uso do design como um meio de satisfazer as necessidades humanas. 
Brown e Wyatt (2010) afirmam que os designers tradicionalmente focaram-se em melhorar a aparência e a funcionalidade dos produtos; contudo, recentemente eles começaram a utilizar ferramentas de design para resolver problemas complexos, originando a abordagem intitulada Design Thinking (Brown \& Wyatt, 2010).

Com relação à sistematização de métodos e técnicas de Design Thinking, os autores Vianna (2012) destacam que são geralmente realizadas por equipes interdisciplinares, divididas em três grandes fases: imersão, ideação e prototipação. Dada a temática do estudo, será abordada a fase de imersão, em que realizasse a observação e a interação de um determinado grupo de pessoas (Brown \& Wyatt, 2010), priorizando-se a qualidade e não a quantidade da amostra. Outro aspecto relevante é a escolha dos usuários, que irão expor suas opiniões, atitudes e experiências a respeito do fenômeno. As informações disponibilizadas pelos usuários irão auxiliar na compreensão dos problemas que poderão conduzir à concepção de novos produtos ou serviços.

Dentre as técnicas utilizadas na fase de imersão do Design Thinking, destacam-se o mapa de empatia e a jornada do usuário. Gibbons (2018a) relata que o mapa de empatia é um dos primeiros passos no Design Thinking, pois possibilita a visualização das atitudes e comportamentos do usuário capazes de auxiliar as equipes a reconhecerem suas necessidades. Ainda, o mapa de empatia revela lacunas nos dados dos usuários e podem ser gerados a partir de entrevistas (Gibbons, 2018a). O mapa de empatia pode ser criado por meio de quatro quadrantes, a fim de responder os seguintes questionamentos:

- o que o usuário sente, nessa reflexão propicia-se percepções emocionais que desencadeiam da questão pesquisada e vivenciada;

- o que o usuário pensa, nessa reflexão evidencia-se o entendimento sobre as principais preocupações e aspirações, necessidades informacionais, pensamentos que mantém sua mente ocupada diante da problemática de pesquisa;

- o que o usuário fala, nessa reflexão fundamenta-se sobre as explanações de ideias e de raciocínios sobre as indagações de pesquisa;

- o que usuário faz, nessa reflexão compreende-se as suas atitudes e seus comportamentos para lidar com o problema de pesquisa.

O mapa de jornada do usuário é uma visualização das experiências que as pessoas têm quando elas interagem com algum produto ou serviço (Hanington \& Martin, 2019; Gibbons, 2018b). As jornadas contam uma história documentada sobre as ações e percepções de um sujeito à medida que ele interage com um produto ou serviço durante um período de tempo (Hanington \& Martin, 2019). A maioria dos mapas de jornada possuem um formato similar (Gibbons, 2018b): no topo, são definidos um usuário e cenário específicos, bem como as expectativas ou objetivos; no centro, são inseridas ações, pensamentos e emoções do usuário; e por fim, na parte inferior, são definidas as oportunidades e insights, como pode ser observado na Figura 1. 
Figura 1: Mapa da jornada do usuário e/ou consumidor/

CUSTOMER/USER JOURNEY MAP

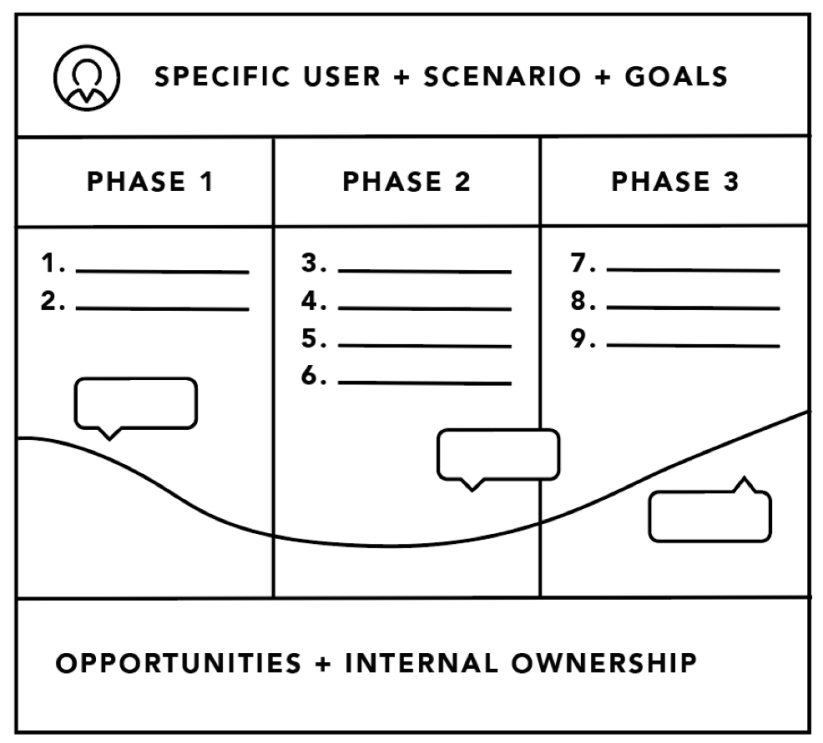

NNGROUP.COM N N/g

Fonte: NNGroup (2018). Disponível em: <https://www.nngroup.com/articles/journeymapping-101/>. Acesso em: 20 set. 2020.

Por meio de entrevistas e criação de mapas de empatia, foi possível sintetizar a jornada do usuário "de candidato a aluno", a fim de atingir a fase de imersão do Design Thinking. Os procedimentos metodológicos são detalhados a seguir.

\section{Procedimentos metodológicos}

A pesquisa de natureza exploratória e carácter qualitativo, utilizou como método a entrevista qualitativa de forma semiestruturada, definida pelas seguintes características (Mason, 2017): I) a troca interacional de diálogo, (II) o estilo informal, (III) a definição de um tema ou tópico central e (IV) a perspectiva de que o conhecimento é situado e contextual. As entrevistas foram realizadas com dez informantes, sendo eles cinco coordenadores de diferentes PPGs em Design e cinco candidatos que participaram de processos seletivos de PPGs em Design.

As entrevistas com os coordenadores de curso tiveram como objetivo: (I) identificar como ocorre a comunicação e divulgação dos editais; (II) apontar os maiores fatores de reprovação dos candidatos nos processos seletivos; (III) obter dicas sobre a preparação para um processo seletivo; (IV) expressar pontos negativos e positivos de um serviço que centralize informações sobre os programas de pós-graduação em Design. Todos os informantes são coordenadores de programas de pós-graduação stricto sensu em Design reconhecido pela CAPES. Dentre os entrevistados, um deles é representante geral do Fórum Nacional de Coordenadores de PPGs em Design, o qual explicou que este Fórum facilita a comunicação via um grupo de WhatsApp 
com vários coordenadores que trocam experiências, notícias, pesquisas e informações entre as Instituições de Ensino.

Já as entrevistas com os candidatos tiveram como objetivo: (I) investigar os motivos que os levaram a optar por cursar uma pós-graduação; (II) identificar como ocorre a busca de informações sobre os processos seletivos; (III) apontar dificuldades durante a preparação para o processo seletivo; (IV) investigar como o processo seletivo poderia ser facilitado e, por fim, criar um mapa de empatia do candidato. Os candidatos entrevistados são oriundos de cinco estados diferentes do Brasil (Amazonas, Distrito Federal, Maranhão, Rio Grande do Sul e São Paulo), tendo participado de um ou mais processos seletivos para um PPG em Design. Dos cinco entrevistados, quatro realizaram o processo seletivo para o nível mestrado. $O$ mapa de empatia foi utilizado com o intuito de compreender as necessidades dos candidatos das quais eles mesmos poderiam não estarem cientes (Gibbons, 2018a), auxiliando na criação da jornada dos candidatos em um processo seletivo para um PPG em Design.

A fim de verificar o roteiro previamente elaborado, foi realizado um teste piloto, no dia $15 \mathrm{de}$ novembro de 2020 , com um discente participante do laboratório ao qual um dos pesquisadores está vinculado. Com o teste, foi possível analisar a pertinência do roteiro e o tempo médio de duração das entrevistas.

As entrevistas foram realizadas pelo Google Meet, entre o período de 20 de setembro a 25 de novembro de 2020, nos horários acordados com os informantes em contato prévio. As dez entrevistas foram conduzidas da seguinte forma: primeiramente, foi explicitado como o método seria conduzido; posteriormente, foi enviado o Termo de Consentimento Livre e Esclarecido por e-mail para assinatura, bem como foi explicitado que a entrevista seria registrada em áudio e vídeo. As entrevistas tiveram cerca de 40 minutos de duração e foram gravadas e transcritas para a análise posterior.

As entrevistas foram analisadas por meio da análise temática (Braun \& Clark, 2012), um método acessível, flexível e cada vez mais popular de análise qualitativa de dados. Braun e Clark (2012, p. 57, tradução nossa) relatam que a análise temática é um método utilizado para "identificar, organizar e oferecer uma visão sistemática dos padrões de significado (temas) em um conjunto de dados." Por ter como foco o significado de um conjunto de dados, a análise temática permite que o pesquisador veja e dê sentido a significados e experiências coletivas ou compartilhadas. Consequentemente, o método é uma ferramenta para identificar o que é comum na maneira como um tópico é falado ou escrito, atribuindo sentido a essas semelhanças.

Braun e Clark (2012) relatam que algumas fases da análise temática são semelhantes às fases de outras pesquisas qualitativas, portanto as etapas não são exclusivas da análise temática. O processo da análise inicia quando o pesquisador começa a notar e procurar padrões de significado e questões de interesse potencial nos dados (Braun \& Clark, 2012). Logo, o objetivo é a apresentação do conteúdo e do significado de padrões nos dados, que irão originar os temas. Esses, por sua vez, são identificados pelo pesquisador antes, durante e depois da análise. 


\section{Resultados e discussões}

A seguir, são apresentados os resultados das pesquisas com os coordenadores e candidatos aos PPGs. As últimas, por sua vez, auxiliaram na construção dos instrumentos de Design

Thinking, sendo eles o mapa de empatia e o mapa da jornada do candidato.

\section{Coordenadores de curso}

As entrevistas semiestruturadas aplicadas aos coordenadores evidenciaram com profundidade as percepções de quem avalia e organiza os processos seletivos de pós-graduação. No Quadro 1, foram sistematizados os comentários mais recorrentes durante a entrevista com os coordenadores.

Quadro 1: Síntese das entrevistas com os coordenadores.

TEMAS

COORDENADORES

\begin{tabular}{|c|c|c|c|c|c|}
\hline Meios e mídias para divulgar os processos seletivos & $\mathrm{C} 01$ & $\mathrm{CO2}$ & $\mathrm{CO3}$ & $\mathrm{CO4}$ & $\mathrm{CO5}$ \\
\hline Edital disponibilizado no site da instituição & $\mathrm{x}$ & $\mathrm{x}$ & $\mathrm{x}$ & $\mathrm{x}$ & $\mathrm{x}$ \\
\hline Divulgação nas redes sociais do PPG & & & & $x$ & $\mathrm{x}$ \\
\hline Suporte de apoio ou preparação para os candidatos & $\mathrm{C01}$ & $\mathrm{CO2}$ & $\mathrm{CO3}$ & $\mathrm{CO4}$ & $\mathrm{C05}$ \\
\hline Modelo para o projeto de pesquisa & $x$ & $x$ & & & \\
\hline Contato com a secretaria do PPG ou e-mail dos professores & & $x$ & $x$ & & \\
\hline Roteiro do projeto, conforme divulgado no edital & & & $x$ & $x$ & $x$ \\
\hline Cursos preparatórios para auxiliar na escrita do projeto & & & & $x$ & $x$ \\
\hline Possíveis fatores de aprovação dos candidatos & $\mathrm{C} 01$ & $\mathrm{CO2}$ & $\mathrm{CO3}$ & $\mathrm{CO4}$ & $\mathrm{C} 05$ \\
\hline Qualificar o problema de pesquisa e suas percepções & $x$ & & & $x$ & \\
\hline Identificação com a carreira acadêmica & & $\mathrm{x}$ & $x$ & & \\
\hline $\begin{array}{l}\text { Compatibilidade das habilidades curriculares do Lattes com as linhas } \\
\text { de pesquisa }\end{array}$ & & $\mathrm{x}$ & & & \\
\hline Projeto de pesquisa viável com as linhas de pesquisa do programa & & & $\mathrm{x}$ & & \\
\hline Alinhamento da pesquisa com o programa e com o orientador & & & $x$ & $x$ & $x$ \\
\hline Desempenho na prova geral de conhecimentos em Design & & & & $x$ & \\
\hline Performance na entrevista & & & & & $x$ \\
\hline Possíveis fatores de reprovação dos candidatos & $\mathrm{C} 01$ & $\mathrm{CO2}$ & $\mathrm{CO3}$ & $\mathrm{CO4}$ & $\mathrm{C05}$ \\
\hline Problemas na escrita e construção textual do projeto de pesquisa & $x$ & $\mathrm{x}$ & & & \\
\hline Entrega incompleta de documentos & & & & & $x$ \\
\hline Ausências nas etapas do processo seletivo & & & & & $x$ \\
\hline Generalizações na pesquisa e projetos não fundamentados & & & & & $x$ \\
\hline Pontos positivos da plataforma & $\mathrm{C} 01$ & $\mathrm{CO2}$ & $\mathrm{CO3}$ & $\mathrm{CO4}$ & $\mathrm{C} 05$ \\
\hline Motivação para auxiliar o candidato & $x$ & & & & \\
\hline Manter atualizado a área de pesquisa do professor em vigência & $x$ & & & & \\
\hline Montar bancas e referências de pesquisa & & & $x$ & & \\
\hline Plataforma aberta para livre acesso & & $\mathrm{x}$ & & $\mathrm{x}$ & \\
\hline Geração de conteúdo em forma de vídeos & & & & & $x$ \\
\hline Pontos negativos da plataforma & $\mathrm{C} 01$ & $\mathrm{CO2}$ & $\mathrm{CO3}$ & $\mathrm{CO4}$ & $\mathrm{CO5}$ \\
\hline O uso pode oportunizar uma concorrência entre os programas & $x$ & & & & \\
\hline Sugestões & $\mathrm{C} 01$ & $\mathrm{CO2}$ & $\mathrm{CO3}$ & $\mathrm{CO4}$ & C05 \\
\hline O portal deve ser vinculado aos sites dos programas e/ou a Capes & $x$ & & & & $x$ \\
\hline Informações sobre as cidades e sobre custo de moradias & & $\mathrm{x}$ & & & \\
\hline Inclusão de espaço para críticas e melhorias na plataforma & & & & & $x$ \\
\hline
\end{tabular}


No tema mídias e meios para a divulgação dos processos seletivos, apurou-se uma comunicação muito similar entre os PPGs em Design, concentrando como estratégia de midiatização dos editais os sites da instituição. Apenas duas instituições das cinco entrevistadas já utilizam as redes sociais como meio de divulgação de informações sobre os processos seletivos. Isso ressalta uma oportunidade de uso das redes sociais para estimular novas formas de interação informacional entre os programas e os candidatos.

Em suporte e apoio aos candidatos apontou um padrão de roteiro a ser seguido pelos projetos de pesquisa e iniciativas de auxílio metodológico por meio de cursos oferecidos pelas instituições, evidenciando a importância desse tipo de conteúdo para os alunos que ainda vão tentar Mestrado e Doutorado em Design.

Já em fatores de aprovação e reprovação dos candidatos demonstram convergências em suas respostas tanto sobre o alinhamento do projeto de pesquisa com a linha de pesquisa escolhida quanto sobre a motivação para se tornar um pesquisador. Corroborando para dois nichos informacionais relevantes neste tema: os conteúdos sobre o que é ser um pesquisador e sobre mapeamentos de linhas de pesquisa presente nas pós-graduações em Design.

O último tema potencial codificado nas entrevistas foi o mais subjetivo e trabalhou em cima do tópico sobre os pontos positivos/negativos da ideia da plataforma e sugestões para a proposta. Na visão geral dos entrevistados, a criação de uma plataforma direcionada para a centralização de informações sobre os PPGs em Design seria algo muito positivo, pois ajudaria os candidatos e os professores em diversas instâncias: (I) na aproximação e integração entre as instituições e entre os candidatos/alunos; (II) no auxílio para formação de bancas e troca de referências; e (III) na atualização informacional das pesquisas e projetos em vigências.

Entretanto, foram questionados pelos entrevistados o que essa plataforma ofereceria de diferencial na vida dos candidatos além de organizar as informações espalhadas pela rede. Sugerindo-se a vinculação com os sites institucionais ou com a CAPES; cursos de metodologias de pesquisa; informações e curiosidades sobre as universidades e sobre custo de vida das cidades; e a reiteração sobre a necessidade de informações a respeito do que é ser um pesquisador a nível de Mestrado e Doutorado e as habilidades e desafios presentes na carreira acadêmica.

Após essa imersão nas opiniões, nas expectativas e nas diretrizes criativas dos coordenadores que serão consideradas diante da possível ideia de criação de uma plataforma para auxiliar os candidatos nos processos seletivos, se faz fundamental analisar o público que incorporaria essa proposta na sua jornada de candidato, com entrevistas que imergiram nas dificuldades encontradas em participar desses processos seletivos.

\section{Candidatos}

A síntese das entrevistas com os candidatos pode ser visualizada no Quadro 2, em que foram evidenciados os códigos recorrentes nas respostas dos entrevistados. As entrevistas com os candidatos evidenciaram motivos que os levaram a cursarem uma pós-graduação stricto sensu, sendo a aspiração de seguir carreira acadêmica e tornar-se professor a mais recorrente. 
Entretanto, dois entrevistados apresentaram diferentes perspectivas: o C4 evidenciou a busca por uma titulação consolidada na área de Design e o C5 relatou a necessidade de buscar aprofundamento teórico e compreender outras possibilidades de atuação da profissão designer que não se restrinjam a criação e/ou produção de artefatos. Com relação à busca de informações sobre os PPGs e universidades, grande parte dos entrevistados relatou ter acessado os sites das universidades e PPGs. Outra questão evidenciada nesse tema foi o contato com amigos e/ou conhecidos que já são alunos no PPG, possibilitando que os candidatos obtivessem informações sobre professores, projetos e linhas de pesquisa.

Quadro 2: Síntese das entrevistas com os candidatos.

\begin{tabular}{|c|c|c|c|c|c|}
\hline \multirow{2}{*}{$\begin{array}{l}\text { TEMAS } \\
\text { Por que cursar uma pós-graduação stricto sensu em Design? }\end{array}$} & \multicolumn{5}{|c|}{ CANDIDATOS } \\
\hline & $\mathrm{C} 01$ & $\mathrm{CO2}$ & $\mathrm{CO3}$ & $\mathrm{CO4}$ & $\mathrm{C} 05$ \\
\hline Seguir carreira acadêmica & $\mathrm{x}$ & $x$ & $x$ & & \\
\hline Ser professor & $x$ & $x$ & $x$ & & \\
\hline Ter uma titulação consolidada & & & & $\mathrm{x}$ & \\
\hline Prestar concurso público & & & & $x$ & \\
\hline Aprofundar conhecimento em Design aplicado à questões sociais & & & & & $x$ \\
\hline Onde buscou informações? & $\mathrm{C01}$ & $\mathrm{CO2}$ & $\mathrm{CO3}$ & $\mathrm{CO4}$ & $\mathrm{C05}$ \\
\hline Site de buscadores & $\mathrm{x}$ & $x$ & $x$ & $\mathrm{x}$ & \\
\hline Site das universidades/PPGs & $\mathrm{x}$ & $x$ & $x$ & $x$ & $x$ \\
\hline Conversas com alunos e professores & $\mathrm{x}$ & $x$ & $x$ & & \\
\hline Quais foram as dificuldades e dúvidas? & $\mathrm{C} 01$ & $\mathrm{CO2}$ & $\mathrm{CO3}$ & $\mathrm{CO} 4$ & $\mathrm{C05}$ \\
\hline Como encaixar o que eu pesquiso no PPG? & $x$ & $x$ & & & \\
\hline O que os professores do PPG pesquisam atualmente? & $x$ & & & & \\
\hline Qual o perfil de aluno que o PPG procura? & $\mathrm{x}$ & $x$ & & & \\
\hline Qual programa escolher? & & & $x$ & & \\
\hline Como estruturar o projeto? & & & $x$ & & \\
\hline O que o PPG espera do pré-projeto? Qual nivel de detalhamento? & & & & $\mathrm{x}$ & \\
\hline Falta de apoio, acessibilidade aos materiais de estudo & & & & & $x$ \\
\hline O que poderia facilitar o processo seletivo? & $\mathrm{C} 01$ & $\mathrm{CO2}$ & $\mathrm{CO3}$ & $\mathrm{CO4}$ & $\mathrm{C} 05$ \\
\hline Atualização dos sites do PPGs com as pesquisas atuais & $\mathrm{x}$ & $x$ & & & \\
\hline Contato prévio com professores do PPG & $\mathrm{x}$ & & & $x$ & \\
\hline Isenção de taxas & & $x$ & & & \\
\hline A instituição possuir um modelo padrão para o pré-projeto & & & $\mathrm{x}$ & & \\
\hline Melhor divulgação dos editais & & & & & $x$ \\
\hline Ações que auxiliem o preparo do candidato & & & & & $x$ \\
\hline
\end{tabular}

O tema sobre dificuldades e dúvidas com relação ao processo seletivo foi o que apresentou a maior diversidade de respostas que demonstraram questões pessoais e individualizadas. Por fim, quanto ao último tema, os candidatos ressaltaram questões como a atualização dos sites dos PPGs, a midiatização dos editais, ter contato prévio com professores do PPG e ações que auxiliem o preparo dos candidatos.

As entrevistas permitiram identificar diferentes jornadas dos candidatos aos PPGs. Por meio das respostas, foi possível verificar similaridades de ações e pensamentos durante o preparo para a submissão, o que conduziu à criação do mapa de empatia. 


\section{Mapa de empatia dos candidatos}

Como já explicitado, o mapa de empatia foi utilizado como técnica para auxiliar a compreender as necessidades dos candidatos das quais eles mesmos poderiam não estarem cientes (Gibbons, 2018a). O mapa foi dividido em quatro quadrantes, a fim de identificar o que o usuário pensa, sente, fala e faz, tratando-se do processo de submissão a um programa de pósgraduação. Portanto, o mapa de empatia permitiu que o candidato descreve-se a sua jornada individual, por meio da autorreflexão do seu processo. Ao fim de cada entrevista, o instrumento foi preenchido pelos entrevistados com o auxílio do mediador, totalizando cinco mapas de empatia. Após essa etapa, os mapas foram analisados de modo que permanecessem apenas as indagações e afirmações que possuíam recorrência em dois ou mais instrumentos, com o intuito de gerar um mapa de empatia sintetizador das experiências dos candidatos, disponível na Figura 2.

Figura 2: Mapa de empatia dos candidatos.

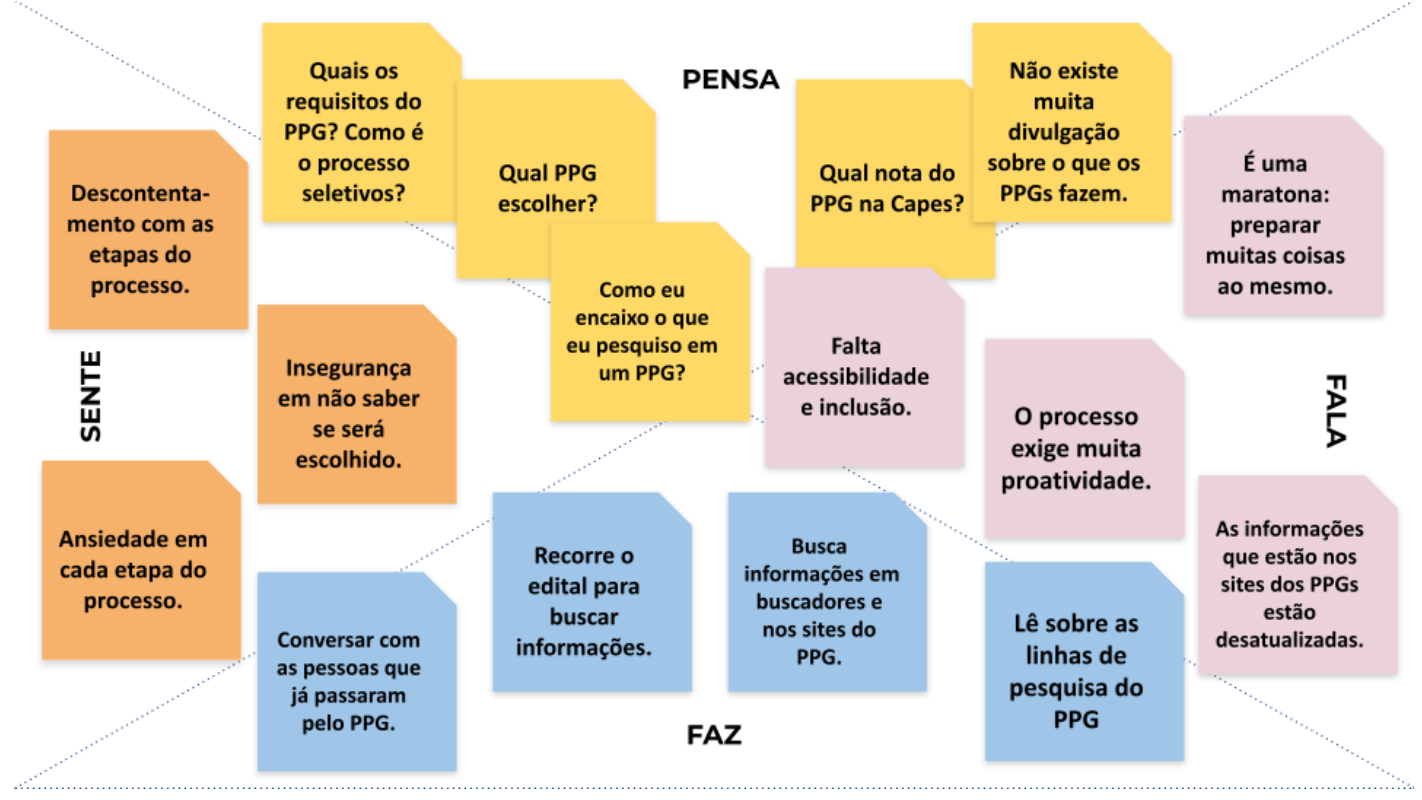

No mapa de empatia, é possível constatar que existem dificuldades quanto à aplicação do candidato em um processo seletivo para a pós-graduação stricto sensu. Embora todos os processos seletivos possuam um edital, por vezes, esses não possuem clareza nas informações, o que dificulta a compreensão de candidatos que estão concorrendo pela primeira vez em um processo desse tipo.

No quadrante "pensa", os candidatos expuseram suas principais indagações sobre necessidades e preocupações durante o processo seletivo. Destacam-se as dúvidas sobre qual PPG escolher e a falta de divulgação sobre as ações dos PPGs. A fim de contornar esse problema, os candidatos recorrem a amigos ou conhecidos que já fazem parte do PPG e buscam informações nos sites dos PPGs, como pode ser identificado no quadrante "faz". 
Outro ponto ressaltado no mapa e nas entrevistas é com relação à falta de acessibilidade e inclusão no processo seletivo, relatados no quadrante "fala": muitos processos são pagos, o que restringe a participação de candidatos em mais de um PPG. Além dos gastos com a taxa de submissão, se o candidato aplicou para outra região, ele precisa arcar com gastos com deslocamento e alimentação para participar das provas e/ou entrevistas que possuem curta duração. Esses gastos também dificultam a aplicação de candidatos para PPGs que sejam muito distantes de seu local de moradia.

A falta de midiatização dos editais foi um ponto presente em três dos cinco mapas dos candidatos, o que evidencia a necessidade de uma maior divulgação dos PPGs em outros locais que não sejam apenas o site do programa ou da universidade.

\section{Mapa da jornada "de candidato a pós-graduando"}

A partir das entrevistas e do mapa de empatia, foi possível criar um mapa da jornada do candidato, por meio da identificação de um cenário e das expectativas do candidato e de suas ações, decisões e questionamentos em cada uma das etapas. A jornada, disponível na Figura 3, foi organizada de forma cronológica e dividida em quatro etapas.

Na coluna (1) definir, foram inseridas as etapas prévias ao processo de submissão a um PPG. Destaca-se nessa etapa a análise do deslocamento para outros PPGs de diferentes cidades e estados, sendo uma definição substancial para dar sequência ao processo seletivo. $O$ candidato deverá avaliar se possui condições financeiras de custear as despesas de deslocamento e alimentação para as etapas do processo seletivo o que, por vezes, o impede de aplicar para PPGs distantes de sua moradia ainda que eles possuam linhas de pesquisa de seu interesse.

Em (2) comparar, é o momento em que o candidato começa o seu processo de decisão entre qual PPG irá aplicar. Nessa etapa, o candidato passa a refletir sobre sua escolha quanto ao PPG, bem como buscar informações sobre os professores das linhas de pesquisa. Nas entrevistas, os candidatos alegaram iniciar a pesquisa por meio de buscadores até redirecionarem-se para os sites dos PPGs.

Já em (3) selecionar, o candidato identifica o PPG que possui mais relação com seus interesses de pesquisa e passa a investigar sobre as linhas de pesquisa, publicações e professores. Por fim, em (4) aplicar, o candidato dedica-se integralmente ao edital do PPG escolhido, a fim de cumprir todos os requisitos para a submissão do projeto em busca da aprovação. Em (5) oportunidades, foram inseridas sugestões de ações que poderiam oportunizar a facilitação de cada uma das etapas da jornada.

A jornada permitiu identificar as dificuldades dos candidatos quanto a busca por informações sobre os PPGs, a definição de um projeto de pesquisa que seja de seu interesse e tenha aderência às linhas de pesquisa dos PPGs, ao identificar também possíveis orientadores e compreender com clareza as informações dos editais. Com a definição dessas problemáticas, considera-se que os processos seletivos de PPGs não são muito acessíveis a grande maioria dos candidatos, pois possuem uma série de limitadores que vão desde a busca inicial por informações sobre os PPGs até fatores econômicos e sociais. 
Figura 3: Mapa da jornada dos candidatos.

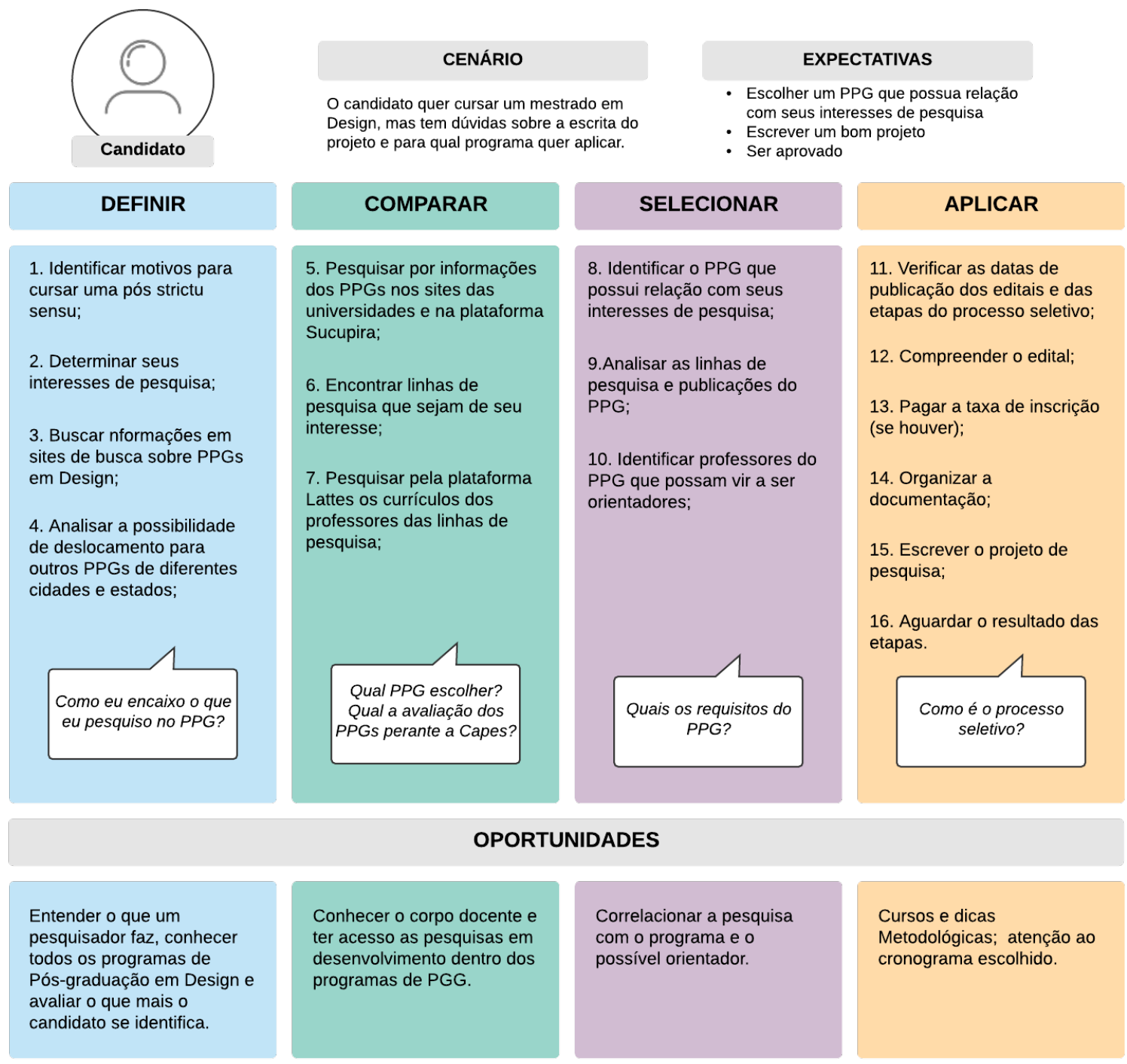

\section{Considerações finais}

Os resultados do estudo destacam que o mapa de empatia permitiu a identificação de fatores emocionais que envolvem a submissão do candidato para um PPG em Design. A fim de materializar esses fatores, foi necessário utilizar uma ferramenta que permitisse a visualização das experiências dos usuários, por isso optou-se pela jornada do usuário. Logo, enquanto o mapa de empatia auxiliou na percepção dos fatores emocionais dos candidatos, com a jornada do usuário foi possível materializá-los em ações. Assim, o objetivo do mapa da jornada "de candidato a pós-graduando" é possibilitar que o candidato encontre direcionamentos sobre como participar de um processo seletivo para um PPG em Design.

Diante das informações levantadas, sintetizadas e analisadas pelas técnicas utilizadas no Design Thinking para imersão sobre as necessidades dos candidatos participantes dos processos seletivos dos PPGs em Design, foi possível identificar a necessidade de um serviço que auxilie na organização dos processos, por meio de ações que amplifiquem a divulgação das 
informações dos processos seletivos. O serviço resultaria em uma plataforma com informações organizadas, centralizadas e atualizadas sobre todos os PPGs em Design no Brasil.

Outro elemento relevante na pesquisa ainda em desenvolvimento, são as sugestões dos coordenadores e as falas dos candidatos nas entrevistas semiestruturadas que quando somadas, transformam-se em orientações para as próximas etapas deste estudo, tais como: (I) mais canais de divulgação dos editais; (II) mapeamento de todos os programas de Design e suas respectivas regiões de atuação; (III) informações sobre o que é ser um pesquisador de mestrado e doutorado; (IV) informações sobre os programas e as suas linhas de pesquisa em vigência; (V) informações sobre custos médio de deslocamento e moradias; e (V) dicas sobre metodologia da pesquisa. Foi evidenciado também que a plataforma pode possibilitar um espaço de debate sobre a necessidade de mais inclusão nos processos seletivos de PPGs.

Por fim, acredita-se que um serviço digital centralizador das informações sobre os PPGs em Design no Brasil irá contribuir para a aproximação de logística, de pesquisa e de orientação de estudantes que desejam seguir carreira como pesquisador em Design. Já os estudos futuros pretendem fundamentar a concepção dessa plataforma, seguindo as próximas etapas metodológicas do Design Thinking até as técnicas finais de prototipação, para assim, colaborar com as muitas jornadas "de alunos a candidatos" de pós-graduação em Design no Brasil.

\section{Agradecimento}

O presente trabalho foi realizado com apoio da Coordenação de Aperfeiçoamento de Pessoal de Nível Superior - Brasil (CAPES) - Código de Financiamento 001.

\section{Referências}

Baer, K., \& Vacarra, J. (2008). Information design workbook: Graphic approaches, solutions, and inspiration +30 case studies. Rockport Publishers.

Bonsiepe, G. (2020). Design, cultura e sociedade. Editora Blucher.

Braun, V., \& Clarke, V. (2012). Thematic analysis. https://doi.org/10.1037/13620-004, 2012.

Brown, T., \& Katz, B. (2011). Change by design. Journal of product innovation management, 28(3), 381-383.

Brown, T., \& Wyatt, J. (2010). Design thinking for social innovation. Development Outreach, 12(1), 29-43.

Coordenação de Aperfeiçoamento de Pessoal de Nível Superior (CAPES). Plataforma Sucupira. (2021). Retrieved 14 May 2021, from https://sucupira.capes.gov.br/sucupira

De Bono, E. (1969). Information processing and new ideas-lateral and vertical thinking. The Journal of Creative Behavior, 3(3), 159-171.

Dick, M. E., Gonçalves, B. S., \& Vitorino, E. V. (2017). Design da informação e competência em informação: relações possíveis | Information design and information literacy: possible relationships. InfoDesign - Revista Brasileira De Design Da Informação, 14(1), 1-13. https://doi.org/10.51358/id.v14i1.500 
Diniz, R. L. (2014). A Pós-Graduação em Design no Brasil: proposta de mestrado oferecida pela Universidade Federal do Maranhão (UFMA). Estudos em Design, 22(3), 57-69.

Gibbons, S. (2018a). Empathy Mapping: The First Step in Design Thinking. Retrieved 14 May 2021, from https://www.nngroup.com/articles/empathy-mapping/.

Gibbons, S. (2018b). Journey Mapping 101. Retrieved 14 May 2021, from https://www.nngroup.com/articles/journey-mapping-101/.

Hanington, B., \& Martin, B. (2019). Universal methods of design expanded and revised: 125 Ways to research complex problems, develop innovative ideas, and design effective solutions. Rockport publishers.

Kelley, T. A. (2001). The art of innovation: Lessons in creativity from IDEO, America's leading design firm (Vol. 10). Broadway Business.

Koberg, D., \& Bagnall, J. (1974). The Universal Traveler, A Soft-Systems Guide to: Creativity, Problem Solving, and the Process of Reaching Goals.[Revised Edition.].

Lipton, R. (2011). The practical guide to information design. John Wiley \& Sons.

Lupton, E. (2011). Graphic design thinking: Beyond brainstorming. Princeton Architectural Press.

Mason, J. (2017). Qualitative researching. Sage.

Nobre, L. N., \& Freitas, R. R. (2017). A evolução da pós-graduação no Brasil: histórico, políticas e avaliação. Brazilian Journal of Production Engineering-BJPE, 26-39.

Osborn, A. (2012). Applied imagination-principles and procedures of creative writing. Read Books Ltd.

Pettersson, R. (2010). It depends. Institutet for Infology.

Triska, R., Vela, J. C., \& Dolzan, J. E. (2014). A pós-graduação stricto sensu do Design no Brasil: uma leitura. Estudos em Design, 22(3), 70-80.

Vianna, M. (2012). Design thinking: inovação em negócios. Design Thinking.

\section{Sobre os autores}

Jéssica R. Esteves, Me., UFSC, Brasil <jessica.rodrigues.esteves@gmail.com>

Larissa B. Ribeiro, Me., UFSC, Brasil <lalibuenano@gmail.com>

Francisco Fialho, Dr., UFSC, Brasil<fapfialho@gmail.com>

Maria C. de Mendonça, Dra., UFPE, Brasil <mariacmendonca@gmail.com>

Richard Perassi, Dr., UFSC, Brasil <richard.perassi@gmail.com>

Berenice S. Gonçalves, Dra., UFSC, Brasil <berenice@cce.ufsc.br>

Ricardo Triska, Dr., UFSC, Brasil <ricardo.triska@ufsc.br> 